\title{
What do international ethics guidelines say in terms of the scope of medical research ethics?
}

\author{
Rosemarie D. L. C. Bernabe*, Ghislaine J. M. W. van Thiel and Johannes J. M. van Delden
}

\begin{abstract}
Background: In research ethics, the most basic question would always be, "which is an ethical issue, which is not?" Interestingly, depending on which ethics guideline we consult, we may have various answers to this question. Though we already have several international ethics guidelines for biomedical research involving human participants, ironically, we do not have a harmonized document which tells us what these various guidelines say and shows us the areas of consensus (or lack thereof). In this manuscript, we attempted to do just that.

Methods: We extracted the imperatives from five internationally-known ethics guidelines and took note where the imperatives came from. In doing so, we gathered data on how many guidelines support a specific imperative.

Results: We found that there is no consensus on the majority of the imperatives and that in only $8.2 \%$ of the imperatives were there at least moderate consensus (i.e., consensus of at least 3 of the 5 ethics guidelines). Of the 12 clusters (Basic Principles; Research Collaboration; Social Value; Scientific Validity; Participant Selection; Favorable Benefit/ Risk Ratio; Independent Review; Informed Consent; Respect for Participants; Publication and Registration; Regulatory Sanctions; and Justified Research on the Vulnerable Population), Informed Consent has the highest level of consensus and Research Collaboration and Regulatory Sanctions have the least.

Conclusion: There was a lack of consensus in the majority of imperatives from the five internationally-known ethics guidelines. This may be partly explained by the differences among the guidelines in terms of their levels of specification as well as conceptual/ideological differences.
\end{abstract}

Keywords: Ethics guidelines, Research ethics, Comparison of guidelines

\section{Background}

When doing research ethics, the basic question is always, "which is an ethical issue and which is not?" It is interesting that though existing internationally-known ethics guidelines on human research somehow help, we cannot help but share Hussein and Upshur's observations, "There are many structural similarities to these varied guidelines, but, more importantly, there is considerable variability and lack of harmonization across the globe" [1]. Up to the present, we know of no literature that attempts to look at the imperatives (i.e., the "oughts") in the major internationally-known ethics

\footnotetext{
* Correspondence: r_bernabe@yahoo.com

Julius Center for Health Sciences and Primary Care, University Medical Center Utrecht, Huispost Str. 6.131, Heidelberglaan 100, 3584 CX Utrecht, The Netherlands

guidelines to allow us some glimpse at what these "structural similarities" are and what areas are lacking in harmonization. The most commonly cited document is Emanuel, Wendler, and Grady's "An ethical framework for biomedical ethics" [2]. Some sources mistakenly perceive this literature as a "coalescence of current standards in a single source" [3]. If we look at Emanuel, Wendler, and Grady's article, there are clear indications that though the various guidelines were consulted, the aim of the article is to produce a "broader, systematic, and comprehensive framework" precisely because for them, existing guidelines are neither systematic nor comprehensive enough [2]. Hence, the Emanuel, Wendler, and Grady framework is just that, another framework, i.e., a proposed ethical structure or system that takes into consideration the various imperatives of the various 
guidelines. The eight principles, i.e., the fundamental truths, of their framework, "are conceptually included in most of the previously mentioned guidance, although existing guidelines do not necessarily include all of them" [2]. As such, there is still the need to comprehensively look into what exactly these international ethical guidelines say on what constitutes ethical issues in human research. Doing so would provide us a glimpse of areas that are widely similar, and hence areas where consensus is present among the international guidelines, and areas where dissimilarities are present, i.e., areas where consensus is lacking. Hence, we not only gain a comprehensive picture, we also get a glimpse of the work that needs to be done in reaching a consensus in research ethics.

\section{Methods}

When choosing the research ethics guidelines to include in this manuscript, we consulted the list of ethics guidelines discussed in section 2, "Codes, declarations, and other ethical guidance for research with humans," of the Oxford Textbook of Clinical Research Ethics [4]. Documents that may provide some ethical guidance but are not necessarily ethical guidelines were excluded, such as the International Conference on Harmonisation Guideline for Good Clinical Practice [5]. This meant that we included five landmark internationally-known ethics guidelines on biomedical research namely, the Nuremberg Code [6], the Declaration of Helsinki [7], the CIOMS International Ethical Guidelines for Biomedical Research Involving Human Subjects (including the explanatory notes) [8], the Council of Europe's Additional Protocol to the Convention on Human Rights and Biomedicine concerning Biomedical Research (including the explanatory notes) [9], and the Belmont Report. [10] From each guideline, we extracted the imperatives. To cluster these imperatives, we used the ethical framework for biomedical research of Emanuel, Wendler, and Grady. Their framework is made up of principles and benchmarks. Benchmarks refer to the imperatives they used as points of reference or measurement of the principles. We used their framework to either check if an imperative is already stated in their benchmarks, to add the imperative under one of their principles, or to add the imperative in a new cluster. It is important to note that since we used Emanuel et al.'s framework as a heuristic tool, we used their principles not as our assumed fundamental truths but as umbrella headings (which we shall call "clusters") for the imperatives. We took the liberty to add new clusters as necessary and to minimally rephrase a principle to allow for wider coverage. For example, instead of "collaborative partnerships" we used "research collaboration" and instead of "fair participant selection," we used "participant selection." Apart from categorizing the imperatives, we also took note where the imperatives came from, and thus we also gathered the data on how many guidelines support a specific imperative, i.e., if there is a strong consensus among the guidelines (SC; 5/5, 4/5), moderate consensus (MC; 3/5), weak consensus (WC; $2 / 5$ ), no consensus (NC; $1 / 5)$, or if it is a non-guideline-based imperative (NGI; 0/5 [in the case of benchmarks in the Emanuel et al. framework that do not correspond to any of the imperatives from any of the guidelines]).

\section{Results}

In total, we extracted 560 imperatives. We included the imperatives for vulnerable population in general, for persons unable to consent, and for emergency research. After double checking for redundancies and merging similar imperatives, the imperatives were reduced to 386. In total, we have 12 clusters: basic principles ${ }^{1}$; research collaboration; social value; scientific validity; fair participant selection; favorable benefit/risk ratio; independent review; informed consent; respect for participants; publication and registration; regulatory sanctions; and justified research on the vulnerable population. Table 1 shows the imperatives within these clusters and their consensus levels. The latter three clusters were added from the original eight principles of Emanuel, Wendler, and Grady. In the following, we describe the clusters and the imperatives. Note that in a few instances, we reclustered some of the Emanuel et al. benchmarks. Additional file 1 gives an overview of the reclustered benchmarks of Emanuel et al.

Of the 386 imperatives, we see in Table 2 that there is the predominance of the lack of consensus at $72.8 \%$. SC and $\mathrm{MC}$ imperatives comprise $8.2 \%$ of all the imperatives. Among all the clusters, Informed Consent has the most number of $\mathrm{SC}$ and $\mathrm{MC}$ imperatives put together, comprising 14 of the 32 (43.8\%). The following clusters have no SC or MC imperatives: the basic principles; research collaboration; social value; and publication and registration; and regulatory sanctions.

\section{Clusters with at least MC}

Table 3 provides a snapshot of the clusters and the corresponding 11 imperatives with SC. In detail, the clusters with at least $\mathrm{MC}$ are the following:

1. Scientific validity The imperatives may be subclustered as follows: scientific design, protocol, professionalism, use of comparator, issues with placebo, reexamination. There is SC that scientific validity entails that the scientific design and the statistical methods of a study must satisfy generally accepted standards and that using these methods and design, the research objectives would likely be met. It also entails that 
Table 1 Ethical imperatives and their levels of consensus (Strong Consensus [SC] 4 or 5/5; Moderate Consensus [MC] 3/5; Weak Consensus [WC] 2/5; No Consensus [NC] 1/5; Non Guideline-based Imperative [NGI])

I Comparison of the basic principles (preambles)

1. The interests and welfare of the human being participating in research shall prevail over the sole interest of society or science. WC

2. Respect for persons (autonomy of autonomous agents and protection for those who lack autonomy) WC respect for persons (autonomy for autonomous agents and protection for those who lack autonomy)

3. Beneficence WC

\subsection{Do no harm WC}

3.2. Maximize possible benefits and minimize possible harms WC

4. Justice as fairness in distribution or what is deserved must be upheld WC

5. It is the duty of the physician to promote and safeguard the health, well-being and rights of patients, including those who are involved in medical research. WC

6. Medical progress is based on research that ultimately must include studies involving human subjects. WC

7. The primary purpose of medical research involving human subjects is to understand the causes, development and effects of diseases and improve preventive, diagnostic and therapeutic interventions WC

8. Medical research should be conducted in a manner that minimizes possible harm to the environment. NC

9. Distinction between therapy and research must be upheld NC

II Research collaboration

1. Involvement of community representatives in planning research, conducting research, disseminating results and use of results to improve health $\mathrm{NGl}$

2. The details of health care services provided to subjects/community/country during and after the trial should be agreed by the sponsor, officials of the host country, other interested parties, and when appropriate, the community from which subjects are drawn. NC

3. Research should be respectful of community's values, circumstances, culture, and social practices. NC

4. Distribution of tangible benefits such as authorship and intellectual property rights must be fair NGl

5. Discussions on responsiveness should include representatives of stakeholders in the host country NC

6. In case of capacity building, the objectives should be determined via a dialogue between external sponsor and host-country NC

7. For equivalency trials, approval should be dependent on the joint negotiations, planning, and justification of the sponsor and health authorities of the host country NC

III Social value

1. Research's social value must be enhanced NG

Responsiveness

2. Research must be responsive to health needs and priorities of host country WC

3. For minor research studies whose outcome is scientific knowledge, there must be assurance that scientific knowledge developed will be for the benefit of the population NC

4. Research should have a potential value for the prospective beneficiaries NC

5. Individuals/groups should benefit from the conduct and the results of the research NGI

Reasonable availability

6. In some instances, drugs should be made available to subjects post-authorization NC

7. Relevant considerations of reasonable availability include the following:

7.1. Length of time intervention will be made available to subjects, community or population NC

7.2. Severity of medical condition NC

7.3. Effect of withdrawing the study drug NC

7.4. Cost to subject or health service NC

7.5. Question of undue inducement NC

Social value in resource-poor settings

8. Research in poor countries whose results are used primarily for the benefit of affluent countries may be characterized as exploitative NC

9. Negotiations on studies in poor countries should include the following:

9.1. Health care infrastructure required NC

9.2. Likelihood of authorization for distribution NC 
Table 1 Ethical imperatives and their levels of consensus (Strong Consensus [SC] 4 or 5/5; Moderate Consensus [MC] 3/5; Weak Consensus [WC] 2/5; No Consensus [NC] 1/5; Non Guideline-based Imperative [NGI]) (Continued)

9.3. Decisions regarding payments, royalties, subsidies, technology and intellectual property; distribution costs NC

10. The development of health-care infrastructure to be used in research and beyond in countries with limited resources must happen at the onset NC

11. For externally sponsored collaborative research where the host country lacks the capacity to assess and ensure scientific quality and/or ethical acceptability, sponsors/investigators must ensure that the research contributes effectively to national or local capacity to design and conduct biomedical research NC

12. In countries with limited capacity of ethical/scientific review, capacity building expected from sponsor dependent on magnitude of research NC

13. Capacity building includes the ff:

13.1. Establishing and strengthening independent and competent ethics review processes and monitoring NC

13.2. Strengthening research capacity NC

13.3. Developing technologies appropriate to health-care and biomedical research NC

13.4. Training of research and health-care staff NC

13.5. Educating the community from which subjects will be drawn NC

14. Obligations of sponsor to provide health care services vary with the circumstances of particular studies and the needs of the host country NC Fair benefits

15. Distribution of fair benefits to the community NGI

IV Scientific validity

Scientific design

1. Scientific design and statistical methods satisfy generally accepted standards and achieve research objectives before approval and throughout research SC

2. Study justified by previous studies and current knowledge SC

3. Research on humans only when there is absence of alternatives WC

4. Research offers means for information not otherwise attainable NC

5. Assessment of scientific quality should take account of the following:

5.1. Research design NC

5.2. Objectives NC

5.3. Technical feasibility NC

5.4. Statistical methods NC

5.5. Potential for reaching valid conclusions with the smallest possible number of participants NC

6. Less invasive procedures should be used once they become available NC

7. Study results must be interpretable and useful in the context of health problem NGI

8. Research design must be feasible given the context NGI

Protocol

9. Design and performance of study must be justified in the protocol WC

10. For multi-center research, any change in the protocol should be made at every collaborating center, or there must be an explicit inter-center comparability procedure NC

Professionalism

11. Research conducted only by scientifically qualified persons. Highest degree of skill and care required through all stages MC

12. Physicians who supervise patients must be competent and qualified NC

13. The current state of the art of scientific knowledge and clinical experience determine the professional standards and skills expected of professionals in the research NC

14. Investigators should not enter into agreements that unduly interfere with their access to data, ability to analyze data independently, to prepare manuscripts, or to publish them NC

Use of comparator

15. Exception to the rule that study drug must be compared with established effective intervention: when established effective intervention is not available and is not likely to be available in the country. NC 
Table 1 Ethical imperatives and their levels of consensus (Strong Consensus [SC] 4 or 5/5; Moderate Consensus [MC] 3/5; Weak Consensus [WC] 2/5; No Consensus [NC] 1/5; Non Guideline-based Imperative [NGI]) (Continued)

16. It is scientifically and ethically preferable to conduct equivalency trials in countries where established effective intervention (as control) is already available. NC

17. For equivalency trials, in the event that established effective intervention is not available and will not be available in the host country, there must be assurances that investigational intervention will be made reasonably available in host country or community NC

18. Studies that do not compare a study drug with an established effective intervention are allowed given the following:

18.1. Responsiveness to health needs of population NC

18.2. When marketing authorization is secured, drug will be reasonably available to the population NC

18.3. Scientific and ethics committees satisfied that the use of established effective intervention would not yield scientifically reliable results relevant to the study population NC

Issues with placebo

19. Placebo may be used given the following:

19.1. No proven intervention exists $M C$

19.2. Participants on placebo not subject to additional risks or serious or irreversible harm WC

19.3. Participants exposed to at most temporary discomfort or delay in relief of symptoms (acceptable risk) WC

19.4. Extreme care to avoid this option NC

19.5. Use of comparator would not yield scientifically NC reliable results

20. Ethical acceptability of placebo trials increases as time decreases and change to active treatment (escape treatment) possible in case of intolerable symptoms NC

21. Trials with placebo that entail only minor risks (even if noninferiority or equivalency trials are possible) may be ethically acceptable given the following:

21.1. REC must be satisfied that the safety and human rights of subjects are fully protected NC

21.2. Participants are fully informed about alternative treatments NC

Reexamination

22. When benefit/risk balance has been disturbed, the trial's continuation should be reconsidered WC

23. In the event of scientific developments, research should be re-examined by a competent body, REC, or DSMB. NC

24. The purposes of re-examination of research project are the following:

24.1. Research needs to be discontinued or if changes are necessary NC

24.2. Research participants or their representatives need to be informed of developments NC

24.3. Additional consent required NC

$\checkmark$ Participant selection

1. Participants are selected to maximize social value and enhance the possibility of benefits to participants NGl

2. Research population is selected to ensure compliance with scientific norms and generate valid and reliable data MC

2.1. Underrepresented groups are given appropriate access to participation WC

2.2. Research should not be offered only to some favored patients (for therapeutic research) or to undesirable persons (for risky research) NC

2.3. The exclusion of groups/communities that might benefit should be ethically justified NC

3. Research population selected to minimize risks to participants NGI

3.1. There should be an order of preference of participants based on ability of members of the class to bear burdens and the appropriateness of placing further burdens on already burdened persons. NC

3.2. For nontherapeutic research with risks, less burdened classes of persons should be called upon first, unless research is directly related to the condition of the more burdened class. NC

4. Groups/communities are selected in such a manner that burdens and benefits are equitably distributed. Deviations from this must be morally justifiable. NC

5. Subjects should be drawn from the qualifying population without prejudice, unless there is a sound scientific reason NC

VI Favorable benefit/risk ratio

Risks

1. Risks and burdens minimized SC 
Table 1 Ethical imperatives and their levels of consensus (Strong Consensus [SC] 4 or 5/5; Moderate Consensus [MC] 3/5; Weak Consensus [WC] 2/5; No Consensus [NC] 1/5; Non Guideline-based Imperative [NGI]) (Continued)

2. Various risks to individual subjects and others (physical, psychological, social, legal) delineated and probability and magnitude quantified to the extent possible MC

3. No unnecessary risks WC

4. Risks monitored, assessed, and documented WC

5. Risks that may affect individuals, families, or society (or a part of society) must be taken into consideration NC

6. Method to ascertain risk explicit NC

7. Information on risk must also cover risks related to individual characteristics of the participants such as age, presence of disorders, among others NC

8. Harm to environment must be minimized NC

Benefits

9. Various possible benefits to individual subjects delineated and probability and magnitude quantified to the extent possible WC

10. Benefits to participants maximized WC

11. Benefits to other individuals or groups affected must be carefully assessed NC

Assessment

12. Risk justified: risk do not outweigh potential benefits (risk to subjects outweighed by anticipated benefit to subject + anticipated benefit to society SC

13. Risk and benefit to subjects are usually considered as carrying special weight NC

14. Interests other than those of the subject may at times justify risks as long as subjects' rights are protected NC

15. In cases of direct benefit, higher risks may be acceptable provided the risks are proportional to benefits NC

16. When assessing direct benefits, a particular course of action must be judged in the light of the participant's specific health problem. NC

17. Therapeutic interventions are justified, in light of benefits and risks, that they will at least be as advantageous to subjects as any available alternative. NC

18. Nontherapeutic interventions justified by expected benefits to society NC

19. Risks of nontherapeutic interventions must be reasonable in relation to knowledge to be gained NC

20. Nontherapeutic research must entail no more than acceptable risk and burden to the participant NC

21. In nontherapeutic research, the level of risk and burden to individuals able to consent may be higher compared to those not able to consent NC

22. For nontherapeutic research, when assessing an intervention, it must meet the criteria of relevance and proportionality between the aim pursued and means employed. NC

23. In assessing risks and benefits of a protocol to a population, it is appropriate to consider the harm that could result by foregoing the research. NC

24. Determine if estimates of risks and benefits are reasonable NC

25. Net risk: knowledge to be gained justifies risk NGI

VII Independent review

Research Ethics Committee (REC) Composition/requirements

1. Independent and competent MC

2. REC provides reasons for decision WC

3. REC members should declare possible conflict of interest WC

4. REC member with interest on a proposal should not take part in assessment WC

5. REC must be multidisciplinary WC

6. Potential conflict of interest, as well as the perception of it, may be as important as actual conflicts. NC

7. Review process transparent NC

8. REC members who withdraw due to conflict of interest should be allowed to offer comments or respond to questions. NC

9. REC members replaced periodically NC

10. Financial assistance should not be provided directly to the REC to avoid conflict of interest and to safeguard the independence of their review. Instead, funds should be made available to appropriate authorities or to the host research institute. NC

11. Participation of laypersons important; the layperson should not be a healthcare professional nor have experience in carrying out biomedical research NC

12. Thought should also be given to gender and cultural balance in the REC composition. NC 
Table 1 Ethical imperatives and their levels of consensus (Strong Consensus [SC] 4 or 5/5; Moderate Consensus [MC] 3/5; Weak Consensus [WC] 2/5; No Consensus [NC] 1/5; Non Guideline-based Imperative [NGI]) (Continued)

Rights and responsibilities of RECS

13. An REC must examine the following:

13.1. Scientific merit: aim study design, safety provisions MC

13.2. risks justified and benefits maximized WC

13.3. if monetary and in-kind recompense constitute undue inducement WC

13.4. IC procedures satisfactory NC

13.5. Subject selection equitable NC

13.6. clear wording of info sheet NC

13.7. when intimidation may be present in securing IC, REC consider whether a neutral 3rd party should seek IC NC

13.8. ethical acceptability of placebo NC

13.9. if procedures are sufficient to verify if subject is capable of consent NC

13.10. In terms of compensation, REC should determine the ff in advance

13.10.1. Injuries for which subjects will receive free treatment and in case of impairment be compensated NC

13.10.2. Injuries for which they will not be compensated NC

14. RECs have the right to report to institutional or governmental authorities any serious or continuing non-compliance. NC

15. Unexpected or unforeseen adverse reactions must be presumed compensable NC

16. When risks are significant, there should be extraordinary insistence by REC on justification NC

For the investigator/sponsor

17. Every interventional study should be submitted to an REC for independent examination and approval. MC

18. At the end, final report with summary of findings and conclusions must be submitted to REC. WC

19. Research project should be submitted to the state where research is to take place. NC

20. All information necessary for ethical assessment shall be provided in writing to the REC. NC

21. The following shall be provided to the REC:

21.1. Info about principal researcher NC

21.2. Aim and justification of research NC

21.3. Methods and procedures including statistical and other analytical techniques NC

21.4. Summary of the project NC

21.5. Statement on previous and concurrent submissions of the project NC

21.6. Justification for involving human beings NC

21.7. Inclusion/exclusion criteria NC

21.8. Reasons for the use or absence of control groups NC

21.9. Nature and degree of foreseeable risks NC

21.10. Nature, extent, and duration of interventions NC

21.11. Arrangements to monitor, evaluate, and react to contingencies NC

21.12. Timing and details of info and the means proposed for the provision of info NC

21.13. Documentation for IC/authorization for participation NC

21.14. Arrangements to ensure privacy and confidentiality NC

21.15. Arrangements for information generated during research which may be relevant to the health of the participant NC

21.16. Details of all payments/rewards NC

21.17. Conflict of interest of researchers NC

21.18. Details of potential further uses, including commercial, of results, data, or biological materials NC

21.19. Other ethical issues perceived by researcher NC

21.20. Insurance or indemnity to cover for damages that may arise during research NC

22. REC may request for additional necessary information NC 
Table 1 Ethical imperatives and their levels of consensus (Strong Consensus [SC] 4 or 5/5; Moderate Consensus [MC] 3/5; Weak Consensus [WC] 2/5; No Consensus [NC] 1/5; Non Guideline-based Imperative [NGl]) (Continued)

23. No protocol amendment w/o REC approval NC

24. Unexpected or unforeseen adverse reactions must be reported to the REC for prompt review as they occur. NC

25. REC should be informed of the reasons for premature termination of a project NC

Externally sponsored studies

26. Ethical standards outside the country of the sponsor should be no less stringent WC

27. Local or national REC reviewing proposals for an external sponsor should have members who are thoroughly familiar with customs and traditions and sensitive to human rights issues. NC

28. Local RECs are usually not authorized to change doses of drugs, inclusion criteria, or similar modifications. NC

29. Changes recommended/demanded by local RECs should be reported to the sponsor for action to ensure protection of other subjects and validity across sites. NC

30. The REC and the health authorities of the host country should verify if the research is responsive to the health needs and priorities of the host country. NC

31. Ethical reviews should be done in both the country of the sponsor and of the host. NC

32. When sponsor is an international organization, review of protocol must be in accordance with its own independent ethical review of standards and procedures. NC

33. RECs within the sponsoring country/international org should determine the following:

33.1. Scientific methods sound and suitable to the aims of the research NC

33.2. Adequate standards of safety NC

33.3. Sound justification for conducting the study in the host country NC

33.4. Research in compliance with ethical standards of the sponsor country/international organization NC

34. When the host country has a developed capacity for independent ethical review, review in the sponsor country may be limited to ensuring compliance with broadly stated ethical standards. NC

35. When host country does not have a developed capacity for review, full review in the sponsoring country or international organization is necessary NC

36. Multiple reviews should be minimized and reconciled NC

When deception/incomplete disclosure is involved

37. When deception is necessary in the research's methodology, the REC should look into the following:

37.1. Consequences for subjects being deceived NC

37.2. Adequate plan for debriefing subjects (whether and how) results NC

37.3. Justification of sponsor that no other research method would suffice NC

37.4. Justification from sponsor that significant advances could result from the research NC

37.5. That nothing has been witheld that, if divulged, would cause a reasonable person to refuse to participate NC

38. In case of research involving incomplete disclosure, the REC should look at the following:

38.1. Incomplete disclosure is truly necessary in the research NC

38.2. No undisclosed risk that is more than minimal NC

38.3. Adequate plan for debriefing subjects and disseminating results to them NC

39. REC should review and approve all proposals to deceive persons other than the subjects NC

40. In cases when other persons (not the subjects) are to be deceived, REC must determine whether these other persons are similarly entitled to the prompt and honest answering of questions NC

41. When subjects cannot be told that information in the IC has been withheld, explicit REC approval necessary NC

Studies affecting groups

42. In studies such as epidemiology or sociology where risks to groups may exist, REC must ensure that interests of all concerned have been given due consideration. NC

VIII Informed consent (IC)

Culture-appropriate consent

1. Disclosure forms and procedures are sensitive to culture, language, context SC

2. Recruitment procedures and incentives are consistent with cultural, political, and social practices NGI 
Table 1 Ethical imperatives and their levels of consensus (Strong Consensus [SC] 4 or 5/5; Moderate Consensus [MC] 3/5; Weak Consensus [WC] 2/5; No Consensus [NC] 1/5; Non Guideline-based Imperative [NGI]) (Continued)

3. Mechanisms to symbolize consent are consistent with culture and context NGl

Securing consent

4. Consent preferably in writing; if not, must be formally documented and witnessed MC

5. IC a process begun with initial contact and continues throughout the course of the study NC

6. Consent process has three elements, all of which must be given importance: information, comprehension, and voluntariness NC Information

7. Potential participants must be informed of the following:

7.1. Anticipated benefits SC

7.2. Right to withdraw without reprisal SC

7.3. Aims MC

7.4. Methods/procedures MC

7.5. Sources of funding $M C$

7.6. Institutional affiliations of researchers MC

7.7. All risks and discomforts that a reasonable person would consider material MC

7.8. Any current alternative interventions MC

7.9. After completion of the study, subjects will at least be given the option to be informed of the findings of the research $M C$

7.10. Post-study provisions WC

7.11. Rights to refuse to participate WC

7.12. After completion of the study, subjects informed of findings that relate to their individual health status WC

7.13. Subjects have rights to access their data on demand, unless otherwise approved by REC WC

7.14. Provisions to ensure privacy and confidentiality WC

7.15. Possible research uses of the subjects medical records and biological specimens taken in the course of clinical care WC

7.16. Whether commercial products may be developed from biological specimens and whether the participant will receive monetary or other benefits WC

7.17. Extent of investigator's/sponsor's responsibility to provide medical services to participants WC

7.18. Explanation of how research differs from routine medical care WC

7.19. Treatment will be provided free of charge for specified types of research-related injuries or complications, nature and duration of such care, who will provide care, and uncertainties if any WC

7.20. Compensation, if any, in case of damage, disability or death WC

7.21. That an REC has approved the protocol WC

7.22. Conflict of interest NC

7.23. Rights and safeguards prescribed by law for the subjects' protection NC

7.24. Rights and safeguards prescribed by law for those not able to consent to research NC

7.25. That the individual is invited to participate NC

7.26. Reasons for considering the individual suitable for the research NC

7.27. Participation is voluntary NC

7.28. For controlled trials, explanation of the features of the research design (e.g., randomization, double-blinding) NC

7.29. Limits to confidentiality and possible consequences of breaches NC

7.30. Policy on use of results of genetic tests and familial genetic info, and precautions to prevent disclosure of results to others without consent. NC

7.31. Sponsors of the research NC

7.32. Whether it is planned that biological specimens collected in the research will be destroyed at its conclusion, and if not, details about storage and possible future use NC

7.33. Subjects have right to decide about the future use of their biological specimens collected during research, to refuse storage, and to have the material destroyed $\mathrm{NC}$

7.34. Whether investigator is only investigator or both investigator and subject's physician NC 
Table 1 Ethical imperatives and their levels of consensus (Strong Consensus [SC] 4 or 5/5; Moderate Consensus [MC] 3/5; Weak Consensus [WC] 2/5; No Consensus [NC] 1/5; Non Guideline-based Imperative [NGI]) (Continued)

7.35. Whether or not compensation is guaranteed in the country NC

7.36. Offering the subject to ask questions NC

7.37. Duration of participation (including number and duration of visits NC

7.38. Possibility of early termination of trial or of participation NC

7.39. Compensation for participation NC

7.40. Prospective subjects need to be informed that they do not need to take legal action to secure free medical treatment or compensation for injury NC

8. Information complete, accurate, and not overwhelming. WC

9. Sometimes, it may be advisable to give info sheets NC

10. IC process should not contain words that absolve the investigator from responsibility in case of accidental injury, or would imply that subjects waive their right to seek compensation for impairment, disability, or handicap NC

11. If a person wishes to not receive detailed information on any are, this should be respected so long as he/she has received sufficient info to give IC NC

12. The wish of a participant to not receive info should be recorded NC

13. When incomplete disclosure/deception is involved, subjects must be asked to consent to remain uninformed of the purpose of some procedures until research is completed WC

14. When incomplete disclosure/deception is involved, participants provided with omitted info after the study NC

Comprehension

15. Participants' comprehension of information must be ensured SC

16. Subjects should have the opportunity to ask questions and receive truthful answers before or during research $\mathrm{MC}$

17. Means must be used to ensure potential participant's understanding of procedure WC

18. Obligation to ascertain comprehension increases with risk NC

Voluntariness

19. The voluntary consent of the participants must be ensured SC

20. IC must be voluntarily obtained for all biomedical research involving humans WC

21. Participants must be actually free to refuse or to withdraw WC

22. Potential participant must be given enough time to decide WC

23. Prospective subject must not be exposed to undue influence (e.g., asking spouse or community leader to influence decision) WC

24. Payments/services should not lead to undue inducement to participate WC

25. Voluntariness demands that consent is free from coercion and undue influence NC

26. In case of a dependent relationship or duress, IC must be sought by a an independent and qualified individual NC

27. Intimidation in any form invalidates IC NC

28. Especially in nontherapeutic research that present more than minimal risk, sponsor/investigator should avoid undue material inducement NC Competence

29. When the capacity of a person to give consent is in doubt, arrangements must be in place to verify this capacity NC

30. For studies where some subjects may be rendered incapable of IC, the initial protocol submitted for approval should anticipate that some patients may be incapable of consent and propose a form of proxy consent NC

Renewing consent

31. Promptly renewing of consent necessary when new information may affect the willingness of a participants, when material changes occur in the conditions or the procedures, and also periodically in long-term studies NC

32. Any new info relevant to participation must be conveyed to research participants or their representatives, if applicable, in a timely manner NC Withdrawal of consent

33. Withdrawal of a patient's consent must be acted on immediately NC

34. In cases when consent is withdrawn but the abrupt discontinuation of therapy could be hazardous to the patient, the healthcare professional must do the following:

34.1. Explain the risk of discontinuing NC

34.2. Seek consent to continue in the study/treatment NC 
Table 1 Ethical imperatives and their levels of consensus (Strong Consensus [SC] 4 or 5/5; Moderate Consensus [MC] 3/5; Weak Consensus [WC] 2/5; No Consensus [NC] 1/5; Non Guideline-based Imperative [NGl]) (Continued)

Person obtaining consent

35. Person obtaining informed consent must be knowledgeable about the research and is capable of answering questions NC

36. The nature of research, needs of potential participant, national practice, and/or law should determine who should provide information to subjects NC

Use of data/specimen

37. IC necessary for research using identifiable human material or data; when consent is impossible or impracticable, permission from REC still necessary. NC

38. Consent forms should include a separate section (or a separate consent form) for subjects who are requested for consent for the use of their biological specimens for research NC

39. Records and specimens of individuals who previously rejected to consent may only be used in public health emergencies NC

40. On secondary use of research records/biological specimens, secondary uses are usually constrained by the conditions specified in the original consent NC

41. It is essential that the original consent process anticipates foreseeable plans for future use NC

42. In terms of consent for secondary use, consent must ask for the following:

42.1. Whether there will be secondary use and whether such use will be limited to the type of study NC

42.2. Conditions under which investigators will be required to contact the research subjects for additional authorization NC

42.3. Investigator's plan, if any, to destroy or to anonymize the records or specimens NC

42.4. The rights of subjects to request destruction or anonymization NC

Waiver of $\mathrm{IC}$

43. Waiver of IC must be considered uncommon and exceptional and must in all cases be approved by REC NC

44. Waiver of signed IC may be granted if:

44.1. No more than minimal risk NC

44.2. Procedures are those for which signed consent forms are not customarily required NC

44.3. Also, when the existence of a signed consent form would be an unjustified threat to the subject's confidentiality NC

45. Waiver of IC may be granted if:

45.1. No more than minimal risk NC

45.2. IC would make the research impracticable NC

46. Consent for the use of medical records/biological specimens may be waived if:

46.1. Research poses minimal risk NC

46.2. Rights/interests of patients not violated NC

46.3. Privacy and confidentiality or anonymity assured NC

46.4. IC would make the study impracticable NC

47. Refusal or reluctance of individuals to participate is not an evidence of impracticability NC

Supplementary consent

48. While supplementary consents or permissions may be obtained, there must be ways to ensure that the individual can still decide independent of spouse or community leader, for example. NC

49. In studies affecting groups (e.g., epidemiology, sociology) where risk to groups may exist, it is advisable supplement IC with community consultation NC

IX Respect for participants

Participant safety

1. Health of participant monitored to minimize harms: One of the functions of the Data and Safety Monitoring Board is to protect subjects from previously unknown adverse reactions or unnecessarily prolonged exposure to inferior therapy MC

2. Criteria for changing dose or procedures for stopping the study for the health of the participants must be adequate WC

3. All reasonable means should be taken to ensure safety (that death or disabling injury will not occur) WC

4. To protect participants from injury, disability or death,

4.1. Adequate health-care facilities provided and incorporated in study for the safe conduct of research WC

4.2. Proper preparations made NC 
Table 1 Ethical imperatives and their levels of consensus (Strong Consensus [SC] 4 or 5/5; Moderate Consensus [MC] 3/5; Weak Consensus [WC] 2/5; No Consensus [NC] 1/5; Non Guideline-based Imperative [NGI]) (Continued)

5. In some research, counseling (about acquiring a disease unless they take precautions) for the participants may be necessary NC

6. When prospective or actual subjects are found to have a disease unrelated to the research or cannot be enrolled because they do not meet health criteria, they must be referred to/be advised to obtain medical care NC

7. Research shall not delay nor deprive participants of medically necessary preventative, diagnostic, or therapeutic procedures. Treatment of patient should not be altered in a detrimental manner to facilitate research. NC

8. Participants assigned to control groups shall be assured of proven methods of intervention available in the country or region. NC

9. To minimize risk, when intervention to be tested is designed to prevent or postpone a lethal or disabling outcome, therapy that is known to be superior to the intervention being tested must not be withheld (unless it can be ethically justified) NC

10. An individual may choose to take part in research a number of times or regularly provided that it does not endanger the participant's health NC

11. All steps taken to assess the state of health of human beings prior to inclusion in research and to ensure that those with increased risk are excluded. NC

12. Where research is undertaken on persons in their reproductive stage, particular consideration must be given to possible adverse impact on current or future pregnancy and the health of the embryo, fetus, or child. NC

Dissemination of research results to participants/community

13. Everyone is entitled to know the information collected about one's health; however, one's wishes to not be informed shall be respected. NC

14. If research gives rise to information relevant to current or future health or quality of life of research participants, this information shall be offered to them within a framework of health care or counseling NC

15. In some circumstances, the right to know or not to know may be restricted for the patient's interest or in order to protect the rights of a third party or a specified public interest NC

16. In the course of a study, sponsors should disclose to proper health authorities information that might be of public health concern. NC

17. There must be sufficient care on the manner of disseminating research results to the participants and the community NC

Privacy/confidentiality

18. Confidentiality procedures must be effectively implemented WC

19. The confidentiality of the subject's research data must be protected WC

20. Everyone has a right to respect for one's private life in relation to information about his/her health NC

Participant care

21. Free and appropriate medical treatment for injuries incurred due to research participation $M C$

22. There must be adequate plans for care for participants after research NC

Compensation

23. There must be appropriate compensation for subjects harmed due to research participation; in case of death, compensation goes to dependents MC

24. Subjects must not be required to demonstrate the investigator's negligence or lack of reasonable skill to claim free medical treatment or compensation. NC

25. A subject who withdraws due to research related reasons (such as unacceptable side-effects) or due to health grounds should be paid in full. NC

26. A subject who withdraws for any other reason other than research or health related should be paid in proportion to the amount of participation NC

27. Subject must not be asked to waive right to compensation NC

28. The payment to a subject who has been removed from a study due to willful noncompliance may be partly or wholly withheld. NC Sociological/epidemiological studies

29. These studies may have the risk of group stigmatization or expose its members to discrimination. The following must be observed:

29.1. Maintain confidentiality during and after the study NC

29.2. Publish resulting data in a manner respectful to all concerned, or in some circumstances not publish at all NC

Physicians and participants

30. Clinical professional who supervises the research should always be accessible to the participants and ready to respond to their health concerns WC

31. Physician involved in research must protect the life, health, dignity, integrity, right to self-determination, privacy, and confidentiality of personal info of research subject NC

32. Physicians who combine research and medical care should involve their patients only if

32.1. This involvement may be justified by the potential value of the research NC 
Table 1 Ethical imperatives and their levels of consensus (Strong Consensus [SC] 4 or 5/5; Moderate Consensus [MC] 3/5; Weak Consensus [WC] 2/5; No Consensus [NC] 1/5; Non Guideline-based Imperative [NGI]) (Continued)

32.2. There is good reason to believe that the health of the patients will not be adversely affected NC

X Publication and Registration

Publication

1. Conflict of interest, sources of funding, and institutional affiliation must be declared in publications WC

2. Publishers should retract any article that has been subsequently found to contain falsified or fabricated data or has been based on unethical research. NC

3. Complete and accurate research results must be publicly available NC

Registration in a database

4. Every research involving human subjects must be registered in a publicly-available database before recruitment NC

XI Regulatory Sanctions

1. Disciplinary sanctions must be used as last resort. Preferred methods include cultivation of atmosphere of mutual trust, education and support to promote the capacity for ethical conduct in research. NC

2. Drug regulatory authorities should consider refusal to accept unethically obtained data submitted in support of a marketing authorization application. However, they should also consider the deprivation of benefit to the intended segment of society. NC

XI Special populations

Vulnerable population in general

1. safeguards in place to protect the vulnerable depending on their circumstance (age, gender, economic deprivation, social marginalization, clinical status, etcetera) from being involved in research solely for admin convenience or because they are easy to manipulate. MC

2. Research must be justified by its responsiveness to health needs/priorities of the group. Group stands to benefit from knowledge, practice, or interventions that result. WC

3. Research on vulnerable population justified only when research of comparable effectiveness cannot be carried out on a non-vulnerable group. WC

4. Justification required in inviting vulnerable individuals as research subjects. WC

5. RECs reviewing proposals directed at specific diseases or impairments or involving vulnerable people should invite representatives or advocates. NC

6. Though socioeconomically vulnerable people should not disproportionately carry the burden of research, they shouldn't also be categorically excluded, especially when research addresses a problem prevalent in the group. NC

7. Overuse of certain groups is unjust. NC

8. Research subjects and other members of vulnerable class must be assured of reasonable access to diagnostic, preventive, or therapeutic products that may become available. NC

Persons not able to consent (including minors)

9. Research on a person without the capacity to consent may be undertaken only if all are met:

9.1. Necessary authorization given specifically in writing by the legal representative, taking into account the individual's previously expressed wishes and objections. SC

9.2. Assent is secured and dissent respected SC

9.3. Research of comparable effectiveness cannot be carried out on individuals capable of consent (i.e., condition necessary characteristic of the research group) MC

9.4. Results of the research must have the potential to produce real and direct benefit NC

9.5. Person undergoing research has been informed of his/her rights and safeguards prescribed by law, unless this person is not in a state to receive info. NC

9.6. An adult not able to consent shall as far as possible take part in the authorization procedure. The opinion of a minor shall be taken into consideration as an increasingly determining factor in proportion to age and degree of maturity. NC

10. Non-therapeutic research may be authorized subject to the following:

10.1. Minimal (low) risk and minimal (low) burden (i.e., medical test standard); any consideration of additional potential benefits of the research shall not be used to justify an increased level of risk or burden WC

10.2. Research aims to contribute through significant improvement in scientific understanding of the individual's condition, disease or disorder NC

11. When risk is above low on researches on individuals unable to consent, the following must be ensured:

11.1. Research is designed to be responsive to disease or conditions they are particularly susceptible to NC

11.2. Risks are only slightly greater than low-risk NC

11.3. Objective of the research sufficiently important to justify exposure of subjects to increased risk NC 
Table 1 Ethical imperatives and their levels of consensus (Strong Consensus [SC] 4 or 5/5; Moderate Consensus [MC] 3/5; Weak Consensus [WC] 2/5; No Consensus [NC] 1/5; Non Guideline-based Imperative [NGI]) (Continued)

11.4. Interventions are reasonably commensurate to the condition under investigation NC

11.5. REC approval NC

12. For studies with individuals unable to consent, such studies may be approved without special substantive or procedural protective measures if the study is low-risk (routine medical tests standard) NC

13. When subjects initially unable to consent becomes capable of consenting, their consent for continued participation must be secured NC

14. Objection to participation, refusal to give authorization or withdrawal of authorization shall not lead to any form of discrimination against the person concerned, in particular regarding the right to medical care. NC

15. Patients with an acute condition that renders them incapable of giving consent may be eligible for inclusion in a trial in which the majority of the prospective subjects will be capable of consent. NC

Third party authorization

16. Third parties are those most likely to understand the incompetent subject's situation and to act in that person's best interest NC

17. Person authorized to act on behalf of a subject should be given an opportunity to observe the research NC

18. The guardian asked to give permission should be offered no recompense other than a refund of travel and related expenses $\mathrm{NC}$

19. For individuals physically or mentally unable to consent, and consent from a legal rep cannot be obtained, study may proceed provided that reasons stated in protocol and REC approved. Consent must be secured asap. NC

Emergency research

20. REC approval must be secured first prior to initiating such a study. WC

21. In emergency research where prior research is not possible, participants, or their representatives if relevant, should be given all relevant information as soon as they are in the state to receive it, and their consent to continued participation should be obtained as soon as is reasonably possible WC

22. The REC and the investigator must set a day when IC must be secured; beyond this day, participation must be discontinued. NC

23. There must be sufficient effort to locate an individual who can give surrogate consent NC

24. Research of comparable effectiveness cannot be carried out in persons in non-emergency situations NC

25. As much as possible, there must be the attempt to identify a population that is likely to develop the condition to be studied for the possibility of securing the IC of these prospective subjects NC

26. Where appropriate, plans to conduct emergency research without prior consent of subjects should be publicized within the community in which it will be carried out NC

27. If acceptability of research is in question, there must be community consultation NC

28. Research should have substantial community support NC

29. Previously expressed objections shall be respected. NC

30. Nontherapeutic emergency research may be justifiable given the following,

30.1. it aims to contribute through significant improvement in the scientific understanding of the individual's condition, disease, or disorder NC

30.2. Minimal risk and minimal burden NC

\section{Pregnant women}

31. Pregnant women should be presumed to be eligible for participation in biomedical research. NC

32. Investigators and ethical review committees should ensure that prospective subjects who are pregnant are adequately informed about the risks and benefits to themselves, their pregnancies, the fetus and their subsequent offspring, and to their fertility. NC

33. Research in this population should be performed only if

33.1. it is relevant to the particular health needs of a pregnant woman or her fetus, or to the health needs of pregnant women in general (broadly understood) NC

33.2. Though the decision about acceptability of risk should be made by the mother as part of the informed consent process, it is desirable in research directed at the health of the fetus to obtain the father's opinion also, when possible. NC

33.3. Special safeguards should be established to prevent undue inducement to pregnant women to participate in research in which interventions hold out the prospect of direct benefit to the fetus NC

33.4. Where fetal abnormality is not recognized as an indication for abortion, pregnant women should not be recruited for research in which there is a realistic basis for concern that fetal abnormality may occur as a consequence of participation as a subject in research. NC

33.5. Investigators should include in protocols on research on pregnant women a plan for monitoring the outcome of the pregnancy with regard to both the health of the woman and the short-term and long-term health of the child. NC 
Table 1 Ethical imperatives and their levels of consensus (Strong Consensus [SC] 4 or 5/5; Moderate Consensus [MC] 3/5; Weak Consensus [WC] 2/5; No Consensus [NC] 1/5; Non Guideline-based Imperative [NGI]) (Continued)

34. Research on a pregnant woman which does not have the potential to produce results of direct benefit to her health, or to that of her embryo, fetus or child after birth, may only be undertaken if the following additional conditions are met:

34.1. the research has the aim of contributing to the ultimate attainment of results capable of conferring benefit to other women in relation to reproduction or to other embryos, fetuses or children, broadly understood NC

34.2. research of comparable effectiveness cannot be carried out on women who are not pregnant NC

34.3. the research entails only minimal risk and minimal burden NC

35. Where research is undertaken on a breastfeeding woman, particular care shall be taken to avoid any adverse impact on the health of the child NC

the study must be justified by previous studies and current knowledge (SC); and that the research is conducted only by scientifically qualified persons in terms of both skill and care for the participants (MC). Lastly, in terms of the acceptability of the use of placebo, there is $\mathrm{MC}$ that it may be used when no proven intervention exists.

2. Participant selection

There is $\mathrm{MC}$ that the research population must be selected to ensure the study's compliance with scientific norms and the generation of valid and reliable data.

3. Favorable benefit/risk ratio

The imperatives may be subclustered as follows: risks, benefits, and assessment.

That risks must be justified in the sense that the risks to participants are outweighed by the anticipated benefits to them and the anticipated benefits to society is a SC imperative that acts as the overarching principle of this cluster. This may mean several things such as the following: there is $\mathrm{SC}$ that risks and burdens must be minimized. Also, to ensure proper assessment, there is $\mathrm{MC}$ that the various risks (whether physical, psychological, social or legal) must be delineated and the probability and magnitude of such risks must be quantified as much as possible.

4. Independent review

By independent review, we refer to the ethical assessment by research ethics committees (RECs; otherwise known as institutional review boards). This cluster may be divided into five subclusters: REC composition and requirements, rights and responsibilities of RECs, responsibilities of the investigator/sponsor, externally sponsored studies, and when deception/incomplete disclosure is involved.

There is MC that an REC must be independent and competent and that it must examine scientific merit by looking at study design and safety provisions. There is also MC that every interventional study must be submitted by the sponsor/investigator to a REC for evaluation and approval.

5. Informed consent

There is a wide range of consensus levels in this cluster, the highest being SC. This cluster includes

Table 2 Summary of clusters and the level of consensus per cluster

\begin{tabular}{|c|c|c|c|c|c|}
\hline Clusters & SC & $\mathrm{MC}$ & WC & NC & $\mathrm{NGl}$ \\
\hline Basic principles (10) & & & 8 & 2 & \\
\hline Research collaboration (7) & & & & 5 & 2 \\
\hline Social value (25) & & & 1 & 21 & 3 \\
\hline Scientific validity (37) & 2 & 2 & 5 & 26 & 2 \\
\hline Fair participant selection (10) & & 1 & 1 & 6 & 2 \\
\hline Favorable benefit/risk ratio (25) & 2 & 1 & 4 & 17 & 1 \\
\hline Independent review (80) & & 3 & 8 & 69 & \\
\hline Informed consent (98) & 5 & 9 & 20 & 62 & 2 \\
\hline Respect for participants (36) & & 3 & 7 & 26 & \\
\hline Publication and registration (4) & & & 1 & 3 & \\
\hline Regulatory sanctions (2) & & & & 2 & \\
\hline Research on vulnerable population (52) & 2 & 2 & 6 & 42 & \\
\hline Total & 11 (2.8 \%) & 21 (5.4 \%) & 61 (15.8\%) & $281(72.8 \%)$ & $12(3.1 \%)$ \\
\hline
\end{tabular}


Table 3 Clusters and imperatives with strong consensus

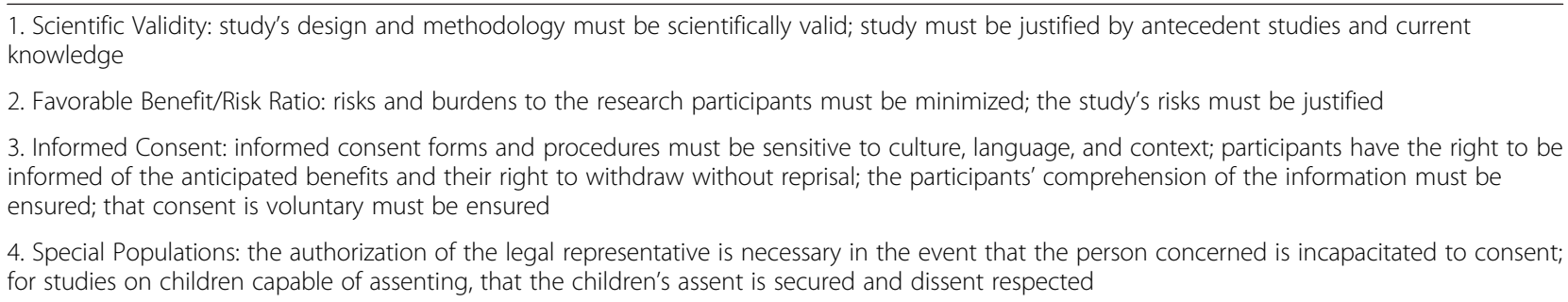

imperatives that relate to an ethical informed consent form and procedure. These imperatives may be subclustered into the following: cultureappropriate consent, securing consent (information, comprehension and voluntariness), renewing consent, withdrawal of consent, use of data/specimen, waiver of IC, and supplementary consent. There is SC that the disclosure forms and procedures must be culturally sensitive. This relates to an $\mathrm{MC}$ that consent must preferably be secured via writing, i.e., through a signature. Otherwise, consent must at least be formally documented and witnessed. In terms of what the participant must be informed about, there is SC that the participant must be informed about the anticipated benefits of the study and the right to withdraw without reprisal. On the other hand, there is $\mathrm{MC}$ that the participant must be informed of the aims; methods/procedures; sources of funding; researchers and their institutional affiliations; all risks and discomforts that a reasonable person would consider material; any current alternative interventions; and that the subjects must at least be given the option to be informed of the findings of the research. In terms of comprehension and voluntariness, there is SC that the investigator must ensure that the participants can in fact comprehend the information that is being provided; and that the participants' consent is truly voluntary.

6. Respect for participants in general

The imperatives may be subclustered as follows: participant safety, privacy/confidentiality, participant care, dissemination of research results to participants/community, compensation, physicians and participants, sociological/epidemiological studies.

There is $\mathrm{MC}$ that the health of the participants must be monitored for purposes of minimizing harm. When injuries are incurred due to research participation, according to a MC imperative, participants have the right to receive free and appropriate medical treatment. Related to this is another MC imperative which states that participants who have been harmed due to research participation must be appropriately compensated and in case of death, the compensation must go to the dependents of the participant.

7. Respect for vulnerable populations The imperatives may be subdivided into the following subclusters: vulnerable population in general, persons not able to consent (including minors), third party authorization, emergency research, and pregnant women.

Research on persons who are not able to consent may be ethically justifiable when the following conditions are met: the authorization is provided in writing by the legal representative who ideally takes into account the participant's prior wishes and objections (SC); assent is secured and dissent respected (SC); and research of comparable effectiveness cannot be carried out on individuals who are capable of giving consent (MC). The following must be in place for the involvement of vulnerable individuals in research to be justified: safeguards must be in place to protect the vulnerable from manipulation or from being involved in a study due solely for administrative convenience $(\mathrm{MC})$.

\section{Clusters with NC}

After looking at the imperatives with a relatively good level of consensus, it is also worthwhile to look at the other end of the spectrum, i.e., clusters with at most NC. These are Research Collaboration and Regulatory Sanctions. The imperatives of these clusters are as follows:

Research collaboration Research must respect the values, circumstances, culture, and social practices of the community. This can be expressed in various ways such as the following: the involvement of community representatives in the planning and conducting research, as well as in the dissemination of results. Also, the details of the health care services information that is distributed to the participants must be the result of an agreement between the sponsor, the host country officials, and when appropriate, the community involved in the research. Discussions on the responsiveness of a research 
must include stakeholder representatives from the host country. Capacity building plans in the host country must be the result of a bilateral dialogue between the host country and the external sponsor. Lastly, authorship and intellectual property rights must be fairly distributed.

Regulatory sanctions The preferred methods for regulatory action must be those that cultivate an atmosphere of mutual trust, education, and support. As such, disciplinary sanctions must be used as the last regulatory resort. Also, drug regulatory authorities should consider refusing to accept unethically obtained data, but not without considering the effect of such a refusal.

\section{Discussion}

In the introduction, we stated that we wanted a comprehensive list of imperatives to determine consensus areas and areas where consensus is lacking. In the results section and in Table 1, as a response to the former, we extensively presented the imperatives according to clusters and subclusters. We also showed the level of consensus present in each cluster. We are now able to determine the areas where consensus is high or at least moderate, and hence the areas of similarities between the guidelines, and the areas where consensus is lacking. From Table 2, we see that of the 386 imperatives, only 32 are at least with MC and only 11 have SC. This means that currently, based on at least MC imperatives, consensus is at $8.2 \%$; and, based on SC imperatives, consensus is at $2.8 \%$.

We also saw that among the imperatives with at least MC consensus, 14 of the 32 come from the Informed Consent cluster. This is also the cluster with the most $\mathrm{SC}$. This may be an indication that there is most agreement within research ethics on informed consent processes and procedures.

At the other end are the clusters Research Collaboration and Regulatory Sanctions where, at most, NC is present. This means not only that there is least agreement within research ethics on these clusters; it also means that since for each entry, only one guideline provides a contribution, these clusters are also the most neglected.

These findings are important for several reasons: first, having a comprehensive picture of the imperatives from the five international ethics guidelines on researches involving humans provides guidance on the question, "which are ethical issues?" In the field of pharmaceutical clinical trial regulation, for example, drug assessors frequently rely on the general statement in the dossier that the clinical trial has been conducted ethically. Given that there is the current trend of increasingly expecting drug regulators to identify and act upon ethical issues [11], having a comprehensive list such as this would help in identifying and categorizing clinical trial issues as ethical in nature. Second, our findings on the level of consensus provide an idea of the magnitude of work to be done in research ethics in terms of international harmonization and agreement. Third, the findings on the clusters where consensus is most present and those where there is no consensus provide some information of which areas are promising in terms of international harmonization and consensus and which areas need more work either because there is no agreement present or because there is relatively little attention on them.

It is important to note that the limited amount of consensus may have been brought about by the fact that some ethics guidelines are more expounded than others. The CIOMS guideline, for example, provides explanatory notes, while the Helsinki Declaration does not. The level of consensus depends on what is written down; however, it may be reasonable to think that some of the low consensus imperatives may in fact be thought of as specifications of the principles stated in shorter guidelines, i.e., that the amount of consensus may increase if only we see some of the low consensus imperatives as specifications of the more general imperatives. There were also differences in terms of the original intended audience of a guideline. The Belmont Report, for example, was originally meant for scientists, members of IRBs, and Federal employees in the USA [10] while the Declaration of Helsinki is addressed primarily to physicians [7]. The latter is the reason why there is NC, for example, on the imperative, "Physician involved in research must protect the life, health, dignity, integrity, right to self-determination, privacy, and confidentiality of personal info of research subject".

At the same time, actual disagreements among the guidelines may also partly explain the limited level of consensus. For our purposes, we shall demonstrate using imperatives from Table 1 that weakness in consensus is in fact reflective of the theoretical differences we see in the literature.

First, we see that on the level of principles, there is at most WC. There is WC that "the interests and welfare of the human being participating in research shall prevail over the sole interest of society or science" and there is also WC that principles such as beneficence and justice must be upheld. This means that, two guidelines subscribe to the former principle, and two other guidelines subscribe to the latter principle. After back checking for sources, suffice it to say that there is no overlap between guidelines on these two camps. The WC is in fact reflective of the wider disagreement within biomedical ethics of what justification entails, i.e., must it be a justification from a moral theory of human rights (i.e., the top-down approach) or must it come from a 
balancing of basic principles (such as the four principles of autonomy, beneficence, justice, and nonmaleficence of Beauchamp and Childress) [12]?

Second, within the cluster Social Value, it is noticeable that both "fair benefits" (i.e., fair distribution of benefits must be determined by the parties involved on a caseby-case basis [13]) and reasonable availability" (i.e., a prior agreement between the trial sponsor and host that the medical product will be made 'reasonably available' to the host country or community once the product has been approved [14]) are present. These two concepts refer to the two differing proposals in the literature on how to make research socially valuable to the host country or community $[14,15]$. Again, this conceptual or ideological difference explains the lack of consensus, with fair benefits being a NGI, and reasonable available as a $\mathrm{NC}$ imperative.

Third, the use of the term "therapeutic research" by imperatives within Table 1 at most garners NC. This is reflective of the discussions in the literature on the acceptability of conflating therapy with research [16-19], i.e., the lack of consensus on any of the imperatives that are hinged on this term is reflective of the disagreement within the literature on the acceptability of therapeutic orientation in research.

The stated differences expounded above in terms of level of detail, intended audience, and actual conceptual/ ideological differences among the guidelines are also unavoidable limitations of this manuscript.

\section{Conclusion}

In this manuscript, we have provided a comprehensive view of what the various international ethics guidelines for research involving human participants say. We also identified the level of consensus among the imperatives from these guidelines and we discovered that in the majority of the imperatives, there is no consensus. Of the 12 clusters, Informed Consent has the highest level of consensus and Research Collaboration and Regulatory Sanctions have the least. This somehow provides a preview of which areas need more work in terms of achieving international harmonization. The lack of consensus may at least be partly explained by the differences among the guidelines in terms of their levels of specification as well as conceptual/ideological differences on which the imperatives are hinged on.

\section{Endnotes}

${ }^{1}$ This cluster contains the explicitly stated "basic principles" (depending on the guideline, this section may be termed as preambles, general ethical principles, and similar phrasings) of the various ethics guidelines.

\section{Additional file}

Additional file 1: An overview of the reclustered benchmarks of Emanuel et al. (DOCX $13 \mathrm{~kb}$ )

\section{Competing interests}

This project was funded by the Dutch Medicines Evaluation Board. The authors have no further competing interests to declare.

\section{Authors' contributions}

RB and GvT designed the study, created the database of ethics imperatives, clustered the entries, interpreted the data, drafted the manuscript, and revised the manuscript. JVD provided inputs in the various versions of the manuscript and have given final approval. All authors read and approved the final manuscript.

\section{Acknowledgement}

This manuscript was written within the context of the Ethics and Clinical Trials Project of the Dutch Medicines Evaluation Board.

Received: 22 April 2015 Accepted: 18 April 2016

Published online: 26 April 2016

\section{References}

1. Hussein G, Upshur R. In: Pinto A, Upshur R, editors. An Introduction to Global Health Ethics. 2012. p. 103-16. Taylor \& Francis.

2. Emanuel E, Wendler D, Grady C. In: The Oxford Textbook of Clinical Research Ethics. Emanuel E, et al., editor. New York: Oxford University; 2008.

3. Kolman JM et al. Conflicts among Multinational Ethical and Scientific Standards for Clinical Trials of Therapeutic Interventions. J Law Med Ethics. 2012;40:99-121.

4. The Oxford Textbook of Clinical Research Ethics. New York: Oxford University; 2008.

5. ICH Harmonised Tripartite Guideline. Guideline for good clinical practice E6(R1). International Conference on Harmonisation of Technical Requirements for Registration of Pharmaceuticals for Human Use; 1996. i-53. http://www.ich.org/fileadmin/Public_Web_Site/ICH_Products/ Guidelines/Efficacy/E6/E6_R1_Guideline.pdf.

6. The Nuremberg code. at <http://www.hhs.gov/ohrp/archive/nurcode.html>

7. World Medical Association. Declaration of Helsinki - ethical principles for medical research involving human subjects. 2013. http://www.wma.net/en/ 30publications/10policies/b3/>

8. Council for International Organizations of Medical Sciences. International Ethical Guidelines for Biomedical Research Involving Human Subjects. CIOMS; 2002. http://www.cioms.ch/publications/guidelines/guidelines_nov_2002_blurb.htm>

9. Council of Europe. Additional protocol to the convention on human rights and biomedicine, concerning biomedical research. 2005. http://conventions. coe.int/Treaty/en/Treaties/Html/195.htm>

10. National Institutes of Health. The Belmont Report. Belmont Rep. Ethical Princ. Guidel. Prot. Hum. Subj. Res. 4-6 (1979). doi:10.1002/9780471462422.eoct093

11. EMA. Reflection paper on ethical and GCP aspects of clinical trials of medicinal products for human use conducted outside of the EU/EEA and submitted in marketing authorization applications to the EU Regulatory Authorities. (2012). at <http://www.ema.europa.eu/docs/en_GB/document_library/Regulatory_ and_procedural_guideline/2012/04/WC500125437.pdf>

12. Beauchamp TL, Childress JF. Principles of biomedical ethics. New York: Oxford University; 2001.

13. Ballantyne A. 'Fair benefits' accounts of exploitation require a normative principle of fairness: response to Gbadegesin and Wendler, and Emanuel et al. Bioethics. 2008;22:239-44.

14. Countries, T. P. in the 2001 C. on E. A. of R. in D. Moral Standards for Research in Developing Countries: From 'Reasonable Availability' to 'Fair Benefits'. Hastings Center Report, vol. 34. 2004.

15. London AJ. In: The Oxford Textbook of Clinical Research Ethics. Emanuel E, et al., editor. New York: Oxford University; 2008. p. 737-744.

16. Miller FG, Rosenstein DL. The therapeutic orientation to clinical trials. N Engl J Med. 2003:348:1383-6.

17. Jansen, L. A. Doctor vs. Scientist? Hastings Cent. Rep. 1 (2008). doi:10.1353/ hcr.2008.0033

18. Bernabe RD, van Thiel GJ, Raaijmakers JA, van Delden JJ. The fiduciary obligation of the physician-researcher in phase IV trials. BMC Med Ethics. 2014;15:11.

19. Anderson JA. Clinical research in context: reexamining the distinction between research and practice. J Med Philos. 2010;35:46-63. 